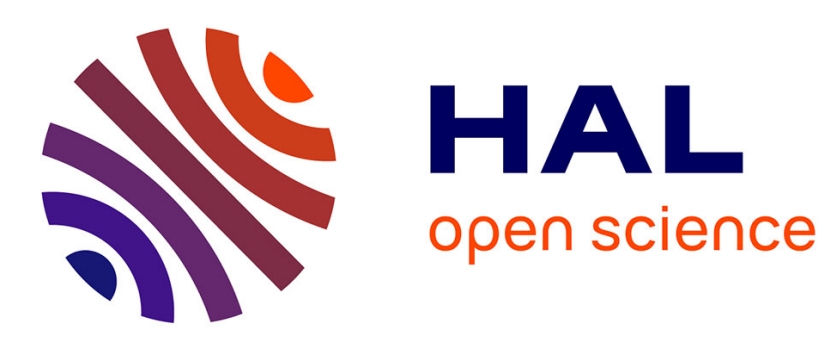

\title{
Analytical examples of diffusive waves generated by a traveling wave
}

Harold Moundoyi, Ayman Moussa, Benoît Perthame, Benoît Sarels

\section{To cite this version:}

Harold Moundoyi, Ayman Moussa, Benoît Perthame, Benoît Sarels. Analytical examples of diffusive waves generated by a traveling wave. Applicable Analysis, 2017, 96 (9), pp.1596-1615. 10.1080/00036811.2017.1314463 . hal-01404972

\section{HAL Id: hal-01404972 https://hal.sorbonne-universite.fr/hal-01404972}

Submitted on 29 Nov 2016

HAL is a multi-disciplinary open access archive for the deposit and dissemination of scientific research documents, whether they are published or not. The documents may come from teaching and research institutions in France or abroad, or from public or private research centers.
L'archive ouverte pluridisciplinaire HAL, est destinée au dépôt et à la diffusion de documents scientifiques de niveau recherche, publiés ou non, émanant des établissements d'enseignement et de recherche français ou étrangers, des laboratoires publics ou privés. 


\title{
Analytical examples of diffusive waves generated by a traveling wave
}

\author{
Harold Moundoyi ${ }^{* \dagger} \quad$ Ayman Moussa $^{\dagger} \quad$ Benoît Perthame $^{\dagger \ddagger} \quad$ Benoit Sarels $^{* \dagger}$
}

November 16, 2016

\begin{abstract}
We construct analytical solutions for a system composed of a reaction-diffusion equation coupled with a purely diffusive equation. The question is to know if the traveling wave solutions of the reaction-diffusion equation can generate a traveling wave for the diffusion equation. Our motivation comes from the calcic wave, generated after fertilization within the egg cell endoplasmic reticulum, and propagating within the egg cell.

We consider both the monostable (Fisher-KPP type) and bistable cases. We use a piecewise linear reaction term so as to build explicit solutions, which leads us to compute exponential tails which exponents are roots of second, third or fourth order polynomials. These rise conditions on the coefficients for existence of a traveling wave of the diffusion equation. The question of positivity and monotonicity is only partially answered.
\end{abstract}

2010 Mathematics Subject Classification. 35C07, 35K57, 35Q92.

Keywords and phrases. Traveling wave; Reaction diffusion; Parabolic systems; Mathematical biology;

\section{Motivations and equations}

Traveling waves are typically generated by the interaction between two physical phenomena: diffusion, or random movement, and reaction, or limited growth. They occur for instance in chemical reactions, ecological invasions, epidemic progressions. In many situations, a traveling wave can interact with purely diffusive species which do not react. A simple question is therefore to know if the wave will generate or not a similar wave in the neutral species. The present work is motivated by one such example arising in developmental biology and related to membrane calcium waves which are observed, after fertilization, through many eukaryotic cells [12, 13. The question which arises, is to know whether the calcic wave which is generated within the egg cell endoplasmic reticulum, can propagate as a wave within the cytoplasm? The counter-intuitive phenomena is that diffusion equations do not have traveling wave solutions and thus it is unclear which phenomena between diffusion induced dispersion or reaction induced wave formation will win, and if a threshold on the coupling strength is necessary.

\footnotetext{
* Sorbonne Universit, UPMC Univ Paris 06, CNRS, UMR 8227, Integrative Biology of Marine Models, Station Biologique de Roscoff, CS 90074, F-29688, Roscoff cedex, France

${ }^{\dagger}$ Sorbonne Universités, UPMC Univ. Paris 06, Laboratoire Jacques-Louis Lions, CNRS UMR7598, F75005 Paris

${ }^{\ddagger}$ Inria de Paris, EPC Mamba
} 
Several studies have been conducted recently about this type of questions when a one dimensional wave is coupled to a two dimension diffusion phenomena, which is the case of calcic waves mentioned above. It also occurs that growth occurs in a two dimensional space with diffusion in a one dimensional (in ecology for instance) [4, 5, 6, 7, 8, 10].

The coupling membrane/volume is also remarkable in the alternative case when traveling waves do not exist and Turing patterns occur [14]. Also remarquable is how non-local diffusion coupled to reaction-diffusion might generate accelerating fronts [1, 2, 15, 16, 17]. Also, notice that the coupling of two reaction-diffusion equation is also of present interest, see [11] and the references therein.

A simpler question is to know how, in a simple one dimensional space, exchanges between a reactiondiffusion equation and a diffusion equation can generate a traveling wave solution thus leading to the formalism

$$
\begin{cases}-c u^{\prime}-u^{\prime \prime}=f(u)+a v, & u(-\infty)=1, \quad u(\infty)=0, \\ -c v^{\prime}-\nu v^{\prime \prime}=b u-(a+d) v, & v(-\infty)=\frac{b}{a+d}, \quad v(\infty)=0,\end{cases}
$$

and, here, the reaction term $f(u)$ represents either a bistable nonlinearity, or a monostable function of Fisher/KPP type [3, 18. The present study proposes a setting where analytical solutions are available therefore leading to explicit calculations. This approach shows very directly, that the existence and uniqueness of a wave speed (or a minimal wave speed in the Fisher/KPP case) faces a much complex algebraic setting that the case of a single equation, which explains the numerous literature on the subject. Depending on the parameters, one can directly understand why different types of solutions may occur.

Notice that the formalism at hand can be seen as a simplified geometric setting of the 2D-1D coupling in case of a band $\Omega=\mathbb{R} \times(0,1)$ coupled to the axis $\{y=0\}$. Indeed, we may depart from the system

$$
\left\{\begin{array}{l}
-c u^{\prime}-u^{\prime \prime}=f(u), \quad u(-\infty)=1, \quad u(\infty)=0, \\
-c w^{\prime}-\nu \Delta w=-d w, \quad \text { in } \Omega, \\
-w_{y}^{\prime}(x, y=0)=b u(x), \quad w_{y}^{\prime}(x, y=1)=0 .
\end{array}\right.
$$

Then, we define

$$
v(x)=\int_{0}^{1} w(x, y) d y
$$

and find after $y$ integration (and because the solution $w$ is in fact independent of $y$ here)

$$
-c v^{\prime}-\nu v^{\prime \prime}=b u-d v, \quad x \in \mathbb{R} .
$$

This reduces the problem (2) to the system (1) with $a=0$.

Our results are organized as follows: we first consider the simple case $a=0$ which explains with very elementary methods how traveling wave solutions arise in the diffusion equation, this is section 2 . We show the surprising result that there are exceptional values of the parameters where the traveling wave cannot be general in the diffusive component. Then, we consider the fully coupled systems in the monostable (section 3) and bistable cases (section 4). The final section presents numerical simulations for the case of interest where the wave propagation is initiated on a circle and the bulk diffusion occurs in the ball. 


\section{The decoupled case}

Setting the problem. As we have motivated above, the simplest example of coupling occurs when, for parameters $b>0, d>0, \nu>0$ and $\theta \in(0,1)$, we consider

$$
\left\{\begin{array}{lc}
-c u^{\prime}-u^{\prime \prime}=f(u), & u(-\infty)=1, \quad u(\infty)=0, \\
-c v^{\prime}-\nu v^{\prime \prime}=b u-d v, & v(-\infty)=\frac{b}{d}, \quad v(\infty)=0 .
\end{array}\right.
$$

Here we consider the specific Fisher/KPP type nonlinearity for which analytical calculations are possible, it is given by

$$
f(u)= \begin{cases}p_{+} u & 0 \leq u \leq \theta \\ p_{-}(1-u) & \theta \leq u \leq 1\end{cases}
$$

with $p_{ \pm}>0$ and the KPP condition at $u=\theta$ which is that $u$ is below its cord at origin and reads

$$
\theta p_{+} \geq(1-\theta) p_{-}
$$

this condition is necessary to enforce $\alpha>0$ in the calculation below.

Obviously the second equation, if uncoupled to $u$, does not have a solution because the heat equation does not admit traveling wave. We will show that, for all values of the parameters $b, d$ and $\nu$ the traveling wave solution $u$ can induce a traveling wave solution $v$.

We recall that for all $c>c^{*}:=2 \sqrt{p_{+}}$there is a traveling wave $u$ and it is given by $\left(\lambda_{1}>0, \mu_{ \pm}>0\right)$

$$
\begin{gathered}
\text { For } x \leq 0, \quad u=1-(1-\theta) e^{\lambda_{1} x}, \quad \text { with } c \lambda_{1}+\lambda_{1}^{2}=p_{-}, \\
\text {For } x \geq 0, \quad u=\alpha \theta e^{-\mu_{-} x}+(1-\alpha) \theta e^{-\mu_{+} x}, \quad \text { with } c \mu_{ \pm}-\mu_{ \pm}^{2}=p_{+} .
\end{gathered}
$$

The parameter $\alpha>0$ is uniquely determined by the continuity of derivatives at 0

$$
(1-\theta) \lambda_{1}=\alpha \theta \mu_{-}+(1-\alpha) \theta \mu_{+} .
$$

We are going to establish the

Theorem 2.1 (Fisher-KPP case, weakly coupled) For all $c>c^{*}:=2 \sqrt{p_{+}}$there exists a unique traveling wave solution of (3) except for three possible values $c_{1}, c_{ \pm}$of $c$ (depending on $b, d, \nu$ and $p_{ \pm}$, these values may be less than $c^{*}$ or even not exist).

For c large, the solution $v$ is not always positive.

The case $c=c^{*}$ requires specific calculations that we do not perform here, but the method can be applied.

The values $c_{1}$ and $c_{ \pm}$can be written explicitly. For instance $c_{1}$ is given, under the condition $(1-\nu)\left(\nu d-p_{-}\right)>0$ by the relation

$$
(1-\nu) c_{1}=\sqrt{c^{2}+4 d}-\sqrt{c^{2}+4 p_{-}} .
$$

Therefore when $p_{+}$and $p_{-}$are small enough, we have $c_{1}>c^{*}=2 \sqrt{p_{+}}$.

Finally, the positivity of the wave is not easy to analyze away from $c \approx+\infty$. We do not know if an additional condition occurs then. We also leave open the question monotonicity.

Proof. We decompose the proof of Theorem 2.1 in several steps. 
Solution for $v$. Now consider the construction of $v$. We are going to introduce five free parameters $\beta_{1}, \beta_{2}, \gamma_{-}, \gamma_{+}$and $\gamma_{2}$

- For $x \leq 0$,

$$
v=\frac{b}{d}-\beta_{1} e^{\lambda_{1} x}-\beta_{2} e^{\lambda_{2} x}
$$

where the $\beta_{2}$ term is the free solution $\left(\lambda_{2}>0\right)$

$$
c \lambda_{2}+\nu \lambda_{2}^{2}=d
$$

The $\beta_{1}$ term is the particular solution

$$
\beta_{1}\left[d-c \lambda_{1}-\nu \lambda_{1}^{2}\right]=b(1-\theta) .
$$

This rises the first condition on $c$, namely that $d-c \lambda_{1}-\nu \lambda_{1}^{2} \neq 0$, and thus the condition $c \neq c_{1}$ in the statement of Theorem 2.1.

- For $x \geq 0$,

$$
v=\gamma_{-} e^{-\mu_{-} x}+\gamma_{+} e^{-\mu_{+} x}+\gamma_{2} e^{-\mu_{2} x}
$$

The free solution term yields the value $\mu_{2}$

$$
-c \mu_{2}+\nu \mu_{2}^{2}=d
$$

The particular solutions gives conditions on the parameters

$$
\begin{gathered}
\gamma_{-}\left[d+c \mu_{-}-\nu \mu_{-}^{2}\right]=\alpha \theta, \\
\gamma_{+}\left[d+c \mu_{+}-\nu \mu_{+}^{2}\right]=(1-\alpha) \theta .
\end{gathered}
$$

These also impose conditions $c \neq c_{-}$and $c \neq c_{+}$.

- For $x=0$, we have to check that $v \in C^{1}$, this means

$$
\begin{gathered}
\frac{b}{d}-\beta_{1}-\beta_{2}=\gamma_{-}+\gamma_{+}+\gamma_{2}, \\
\beta_{1} \lambda_{1}+\beta_{2} \lambda_{2}=\gamma_{-} \mu_{-}+\gamma_{+} \mu_{+}+\gamma_{2} \mu_{2} .
\end{gathered}
$$

Analysis of the algebraic system for the coefficients. Altogether, the conditions (6) - (10) give five equations for five unknowns and we can expect there is a unique solution. We check this now. Except for the values $c_{1}, c_{ \pm}$, we can eliminate directly all variables but $\beta_{2}$ and $\gamma_{2}$. Then, these conditions are reduced to the two conditions

$$
\begin{gathered}
\frac{b}{d}-\frac{b(1-\theta)}{d-c \lambda_{1}-\nu \lambda_{1}^{2}}-\beta_{2}=\frac{\alpha \theta}{d+c \mu_{-}-\nu \mu_{-}^{2}}+\frac{(1-\alpha) \theta}{d+c \mu_{+}-\nu \mu_{+}^{2}}+\gamma_{2}, \\
\lambda_{1} \frac{b(1-\theta)}{d-c \lambda_{1}-\nu \lambda_{1}^{2}}+\lambda_{2} \beta_{2}=\mu_{-} \frac{\alpha \theta}{d+c \mu_{-}-\nu \mu_{-}^{2}}+\mu_{+} \frac{(1-\alpha) \theta}{d+c \mu_{+}-\nu \mu_{+}^{2}}+\gamma_{2} \mu_{2} .
\end{gathered}
$$

These relations are uniquely invertible and give the values $\gamma_{2}$ and $\beta_{2}$

$$
\begin{aligned}
& \lambda_{2} \frac{b}{d}+\left(\lambda_{1}-\lambda_{2}\right) \frac{b(1-\theta)}{d-c \lambda_{1}-\nu \lambda_{1}^{2}}=\frac{\left(\mu_{-}+\lambda_{2}\right) \alpha \theta}{d+c \mu_{-}-\nu \mu_{-}^{2}}+\frac{\left(\mu_{+}+\lambda_{2}\right)(1-\alpha) \theta}{d+c \mu_{+}-\nu \mu_{+}^{2}}+\left(\lambda_{2}+\mu_{2}\right) \gamma_{2}, \\
& -\mu_{2} \frac{b}{d}+\left(\lambda_{1}+\mu_{2}\right) \frac{b(1-\theta)}{d-c \lambda_{1}-\nu \lambda_{1}^{2}}+\left(\lambda_{2}+\mu_{2}\right) \beta_{2}=\frac{\left(\mu_{-}-\mu_{2}\right) \alpha \theta}{d+c \mu_{-}-\nu \mu_{-}^{2}}+\frac{\left(\mu_{+}-\mu_{2}\right)(1-\alpha) \theta}{d+c \mu_{+}-\nu \mu_{+}^{2}} .
\end{aligned}
$$

In other words, except for the possible bad values $c_{1}, c_{ \pm}$of $c$, we have obtained a unique traveling wave solution as announced. 
Positivity for $c \gg 1$. We can perform expansions for $c \gg 1$ and find

$$
\lambda_{1} \approx \frac{p_{-}}{c}, \quad \lambda_{2} \approx \frac{d}{c}, \quad \mu_{-} \approx \frac{p_{+}}{c}, \quad \mu_{+} \approx c, \quad \mu_{2} \approx \frac{c}{\nu} .
$$

We may also compute

$$
\beta_{1} \approx \frac{b(1-\theta)}{d-p_{-}}, \quad \gamma_{-} \approx \frac{\alpha \theta}{d+p_{+}}, \quad \gamma_{+} \approx \frac{1}{c^{2}} \frac{(1-\alpha) \theta}{1-\nu}, \quad \beta_{2} \approx \frac{\theta(b-\alpha)}{d}, \quad \gamma_{2}=O\left(\frac{1}{c^{2}}\right) .
$$

Therefore we may analyze what happens for $x<0$. We observe that the exponential decay is very slow and thus

$$
v \approx(1-\theta) b\left[\frac{1}{d}-\frac{1}{d-p_{-}}\right]+\frac{\theta \alpha}{d},
$$

which is not necessarily positive since $d$ can be chosen as close as $p_{-}$to make the negative term dominant.

\section{Monostable nonlinearity}

For a completely coupled system and a monostable term $f(u)$ we look for $c>0$ large enough to find traveling waves that are solutions of

$$
\left\{\begin{array}{lc}
-c u^{\prime}-u^{\prime \prime}=f(u)+a v, & u(-\infty)=1, \quad u(\infty)=0, \quad u(0)=\theta \\
-c v^{\prime}-\nu v^{\prime \prime}=b u-(a+d) v, & v(-\infty)=\frac{b}{a+d}, \quad v(\infty)=0,
\end{array}\right.
$$

with

$$
f(u)=\left\{\begin{array}{l}
p_{-} u \quad 0 \leq u \leq \theta \\
p_{+}(1-u)-\frac{a b}{a+d} \quad \theta \leq u \leq 1,
\end{array}\right.
$$

Here all the numbers $a, d, p_{ \pm}$are positive and $\theta \in(0,1)$. We assume that it holds:

$$
\nu<1, \quad(1-\theta) p_{+}(a+d)>a b .
$$

For $c$ large enough, we are going to build solutions with the explicit form (here again we implicitly compute with the conditions $\left.\lambda_{0}>0, \lambda_{ \pm}>0, \mu_{ \pm}>0\right)$.

$$
\begin{gathered}
\text { for } x \geq 0 \quad\left\{\begin{array}{l}
u=\alpha_{-} e^{-\lambda_{-} x}+\alpha_{+} e^{-\lambda_{+} x}+\alpha_{0} e^{-\lambda_{0} x}, \\
v=\beta_{-} e^{-\lambda_{-} x}+\beta_{+} e^{-\lambda_{+} x}+\beta_{0} e^{-\lambda_{0} x},
\end{array}\right. \\
\text { for } x \leq 0 \quad\left\{\begin{array}{l}
u=1-\gamma_{+} e^{\mu_{+} x}-\gamma_{-} e^{\mu_{-} x}, \\
v=\frac{b}{a+d}-\delta_{+} e^{\mu_{+} x}-\delta_{-} e^{\mu_{-} x}
\end{array}\right.
\end{gathered}
$$

Theorem 3.1 (Fisher-KPP case) We make the assumption (13) and that $f(\cdot)$ is of Fisher/KPP type, that means (5). Traveling waves with the above expressions and speed $c$ exists if the $\lambda$ 's are the three positive roots of a fourth order polynomial. There is a minimal speed $c^{*}$ such that for $c \geq c^{*}$ these three roots exists.

Asymptotically for $c$ large, the component $u$ is always decreasing and thus there is a traveling wave solution. 
The polynomial under consideration only gives a necessary condition for a traveling wave of the form above. For $c$ not large enough, we do not know if $u$ is monotonic and thus if (11) holds true.

The end of this section is devoted to the proof of Theorem 3.1 .

The exponential form for $x \geq 0$. The construction uses that all solutions are linear combinations of the form

$$
u=\alpha e^{-\lambda x}, \quad v=\beta e^{-\lambda x} \text { for } x \geq 0
$$

satisfies

$$
\left\{\begin{array}{l}
c \lambda-\lambda^{2}-p_{-}=a \frac{\beta}{\alpha}, \\
c \lambda-\nu \lambda^{2}+a+d=b \frac{\alpha}{\beta} .
\end{array}\right.
$$

and thus we look for positive solutions of the fourth order polynomial in $\lambda$

$$
\Pi_{+}(\lambda):=\left(c \lambda-\lambda^{2}-p_{-}\right)\left(c \lambda-\nu \lambda^{2}+a+d\right)=a b .
$$

Notice that, see Fig. 1, for $c>2 \sqrt{p_{-}}$, the above fourth order polynomial $\Pi_{+}(\lambda)$ has one negative root and three positive roots (for $c=2 \sqrt{p_{-}}$a double positive root and another) which satisfy, thanks the assumption $\nu<1$,

$$
\Lambda_{0}:=\frac{c+\sqrt{c^{2}+4 \nu(a+d)}}{2 \nu}>\Lambda_{ \pm}:=\frac{c \pm \sqrt{c^{2}-4 p_{-}}}{2} .
$$

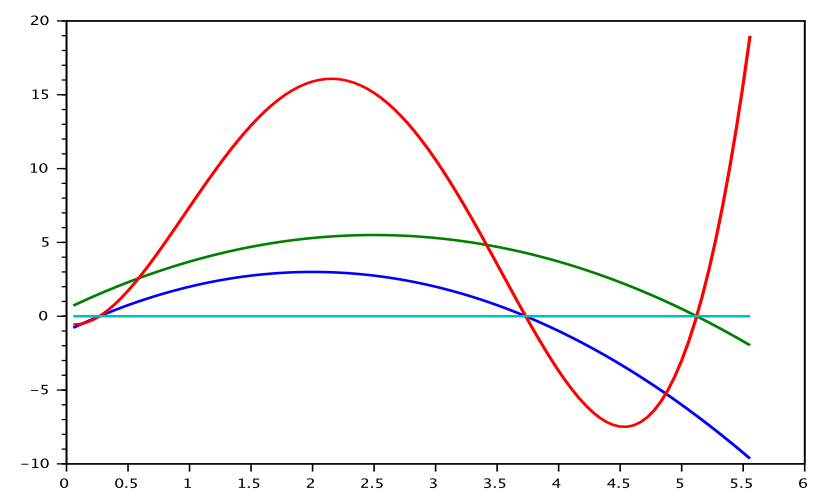

Figure 1: The Fourth order polynomial $\Pi_{+}(\lambda)$ IN RED. The two SECOND ORder POlynomials Which PRoduCt Gives $\Pi_{+}(\lambda)$ ARE Ploted in GReEN (With RoOt $\Lambda_{0}$ ) AND in Blue (With ROOTS $\Lambda_{ \pm}$).

We are going to establish the

Lemma 3.2 We assume (13). There is a value $c^{*}$, with $2 \sqrt{b+p_{-}}>c^{*}>2 \sqrt{p_{-}}$, such that for all $c>c^{*}$, there are three positive solutions of (14) ordered as

$$
\Lambda_{-}(c)<\lambda-(c)<\lambda_{+}(c)<\Lambda_{+}(c)<\Lambda_{0}(c)<\lambda_{0}(c) .
$$

Proof. Firstly, for $\Lambda_{-}<c<\Lambda_{+}$we have

$$
\frac{\partial}{\partial c} \Pi_{+}(\lambda)=\lambda\left[\left(c \lambda-\lambda^{2}-p_{-}\right)+\left(c \lambda-\nu \lambda^{2}+a+d\right)\right]>0
$$


Secondly, for $c^{2}>4\left(b+p_{-}\right)$, at $\lambda=\frac{c}{2}$ we have $\Pi\left(\lambda=\frac{c}{2}\right)>a b$ because it is worth

$$
\left.\Pi\left(\lambda=\frac{c}{2}\right)=\left(\frac{c^{2}}{4}-p_{-}\right)\left[\frac{c^{2}}{4}(2-\nu)+a+d\right)\right]>b(a+d)>a b
$$

and thus we have two positive solutions of (14) in the interval $\Lambda_{-}<c<\Lambda_{+}$.

Consequently, we depart from $c=2 \sqrt{p_{-}}$where there is clearly a single positive solution $\lambda_{0}>\Lambda_{0}$ of (14). When we increase $c$, there is a first value $c^{*}$ where $\max _{\Lambda_{-}<c<\Lambda_{+}} \Pi(\lambda)=a b$ and for $c>c^{*}$ there are two solutions and they are ordered as stated.

We also introduce the notations allowing to recover the $\beta$ 's from the $\alpha$ 's (by $\alpha P=a \beta$ ):

Lemma 3.3 The roots built in Lemma 3.2 satisfy

$$
\partial_{c} \lambda_{-}(c)<0, \quad \partial_{c} \lambda_{+}(c)>0, \quad \partial_{c} \lambda_{0}(c)>0
$$

and for $c \gg 1$

$$
\begin{aligned}
& \left\{\begin{array}{l}
\lambda_{0}(c)=\frac{c}{\nu}+O\left(\frac{1}{c}\right), \\
\lambda_{+}(c)=c\left(1-\frac{p_{-}}{c^{2}}+O\left(\frac{1}{c^{4}}\right)\right), \\
\lambda_{-}(c)=\frac{\rho_{-}}{c}+O\left(\frac{1}{c^{3}}\right), \quad \rho_{-}=\frac{p_{-} a-d+\sqrt{\left(a+d+p_{-}\right)^{2}+4 a b}}{2}>p_{-},
\end{array}\right. \\
& \left\{\begin{array}{l}
0<P_{ \pm}(c):=c \lambda_{ \pm}-\lambda_{ \pm}^{2}-p_{-}<\sqrt{a} b, \quad P_{0}(c):=c \lambda_{0}-\lambda_{0}^{2}-p_{-}<-\sqrt{a} b \\
P_{-}(c)=\rho_{-}-p_{-}+O\left(\frac{1}{c^{2}}\right), \quad P_{+}(c)=O\left(\frac{1}{c^{2}}\right), \quad P_{0}(c) \approx-c^{2} \frac{1-\nu}{\nu^{2}}+O(1) .
\end{array}\right.
\end{aligned}
$$

Proof. Monotonicity in the statement (16). It is a consequence of the equality, obtained differentiating $\Pi_{+}$in $c$ along the roots,

$$
\lambda_{ \pm}\left[\left(c \lambda_{ \pm}-\lambda_{ \pm}^{2}-p_{-}\right)+\left(c \lambda_{ \pm}-\nu \lambda_{ \pm}^{2}+a+d\right)\right]+\partial_{c} \lambda_{ \pm} \Pi_{+}^{\prime}\left(\lambda_{ \pm}\right)=0 .
$$

Indeed, we have $\Pi_{+}^{\prime}\left(\lambda_{-}\right)>0, \Pi_{+}^{\prime}\left(\lambda_{+}\right)<0, \Pi_{+}^{\prime}\left(\lambda_{0}\right)>0$ and it remains to distinguish the signs of the two polynomials which multiplication generates $\Pi_{+}$using

$$
c \lambda_{-}-\lambda_{-}^{2}-p_{-}>0, \quad c \lambda_{+}-\lambda_{+}^{2}-p_{-}>0, \quad c \lambda_{0}-\lambda_{0}^{2}-p_{-}<0 .
$$

The limits at $+\infty$. We notice that, because $\nu<1$,

$$
c \lambda-\lambda^{2}-p_{-}<c \lambda-\nu \lambda^{2}+a+d .
$$

Therefore, for $\lambda_{ \pm}$where these two quantities are positive, we have

$$
a b=\Pi\left(\lambda_{ \pm}\right)>\left(c \lambda_{ \pm}-\lambda_{ \pm}^{2}-p_{-}\right)^{2} \Longrightarrow c \lambda_{ \pm}-\lambda_{ \pm}^{2}-p_{-}<\sqrt{a b} .
$$

And the limits of the $\lambda$ 's follows immediately from this inequality and the explicit roots for equality. Indeed, we find

$$
c \approx \frac{c+\sqrt{c^{2}-4\left(p_{-}+\sqrt{a b}\right)}}{2}<\lambda_{+}(c)<\Lambda_{+}(c) \approx c .
$$

A similar argument gives the result for $\lambda_{0}$. 
Precise expansion for $\lambda_{0}$ in the statement (16). We need however to be more precise and look for the first correction under the form $\lambda_{0}=c R+\frac{s}{c}$. We find

$$
\left(c^{2}\left(R-R^{2}\right)+S-2 S R-p_{-}\right)\left(c^{2} R\left(1-\frac{R}{\nu}\right)+S-2 \nu S R-a-d\right)=a b
$$

which leads to the choice $R=\frac{1}{\nu}, S=-a-d+O\left(c^{-2}\right)$.

Precise expansion for $\lambda_{+}$in the statement (16). Next, we look for the first correction under the form $\lambda_{+}=c\left(1-\frac{R}{c^{2}}+O\left(c^{-4}\right)\right)$. To compute $R$, we insert this form in 14 and find

$$
\begin{gathered}
\left.\left[c^{2}\left(1-\frac{R}{c^{2}}\right)-c^{2}\left(1-2 \frac{R}{c^{2}}\right)-p_{-}+O\left(c^{-2}\right)\right]\left[c^{2}\left(1-\frac{R}{c^{2}}\right)-\nu c^{2}\left(1-2 \frac{R}{c^{2}}\right)+a+d+O\left(\frac{1}{c^{2}}\right)\right)\right]=a b \\
{\left[R-p_{-}+O\left(c^{-2}\right)\right]\left[c^{2}(1-\nu)+O(1)\right]=a b .}
\end{gathered}
$$

Therefore it comes $R=p_{-}$.

Precise expansion for $\lambda_{-}$in the statement (16). We look for an expression $\lambda_{-}=\frac{\rho}{c}+O\left(\frac{1}{c^{3}}\right)$. We find,

$$
\left(\rho-p_{-}+O\left(\frac{1}{c^{2}}\right)\right)\left(\rho+a+d+O\left(\frac{1}{c^{2}}\right)\right)=a b \Longleftrightarrow \rho^{2}+\rho\left(a+d-p_{-}\right)-p_{-}(a+d)-a b=0 .
$$

Statement (17). It follows immediately from inserting the asymptotic expansions for the $\lambda$ 's in the expression for the $P$ 's.

The exponential form for $x \leq 0$. We may proceed similarly for $x \leq 0$ and the corresponding formulas are

$$
\left\{\begin{array}{l}
-c \mu-\mu^{2}+p_{+}=a \frac{\delta}{\gamma}, \\
-c \mu-\nu \mu^{2}+a+d=b \frac{\gamma}{\delta} .
\end{array}\right.
$$

The possible bounded solutions are given by the two roots $0<\mu_{-}(c)<\mu_{+}(c)$ of the polynomial

$$
\Pi_{-}(\mu):=\left(-c \mu-\mu^{2}+p_{+}\right)\left(-c \mu-\nu \mu^{2}+a+d\right)=a b .
$$

These roots satisfy

$$
\left\{\begin{array}{l}
\mu_{-}<\min \left(\frac{-c+\sqrt{c^{2}+4 p_{+}}}{2}, \frac{-c+\sqrt{c^{2}+4 \nu(a+d)}}{2 \nu}\right), \\
\mu_{+}>\max \left(\frac{-c+\sqrt{c^{2}+4 p_{+}}}{2}, \frac{-c+\sqrt{c^{2}+4 \nu(a+d)}}{2 \nu}\right)
\end{array}\right.
$$

and we have

$$
\begin{gathered}
\left\{\begin{array}{c}
\partial_{c} \mu_{-}<0, \quad \partial_{c} \mu_{+}<0, \\
\mu_{ \pm}(+\infty)=\frac{R_{ \pm}}{c}+O\left(\frac{1}{c^{2}}\right), \quad R_{ \pm}=\frac{p_{+}+a+d \pm \sqrt{\left(p_{+}-a-d\right)^{2}+4 a b}}{2}
\end{array}\right. \\
\left\{\begin{array}{l}
Q_{+}(c):=-c \mu_{+}-\mu_{+}^{2}+p_{+}<0, \quad Q_{-}(c):=-c \mu_{-}-\mu_{-}^{2}+p_{+}>0, \\
Q_{ \pm}(+\infty)=-R_{ \pm}+p_{+} .
\end{array}\right.
\end{gathered}
$$

Indeed, on the one hand differentiating $\Pi_{-}$with respect to $c$ gives

$$
-\mu_{ \pm}\left[\left(-c \mu_{ \pm}-\mu_{ \pm}^{2}+p_{+}\right)+\left(-c \mu_{ \pm}-\nu \mu_{ \pm}^{2}+a+d\right)\right]+\partial_{c} \mu_{ \pm} \Pi_{-}^{\prime}\left(\mu_{ \pm}\right)=0 .
$$


On the other hand, we have $\Pi_{-}^{\prime}\left(\mu_{-}\right)<0, \Pi_{-}^{\prime}\left(\mu_{+}\right)>0$ and $Q_{-}(c):=-c \mu_{-}-\mu_{-}^{2}+p_{+}>0$, and $Q_{+}(c):=-c \mu_{+}-\mu_{+}^{2}+p_{+}<0$.

The expansions for $\mu_{ \pm}$are easily obtained as for the $\lambda$ 's, because, keeping the dominant terms in the expression $\mu=\frac{R}{c}$, we find

$$
\left(-R+p_{+}+O\left(\frac{1}{c^{2}}\right)\right)\left(-R+a+d+O\left(\frac{1}{c^{2}}\right)\right)=a b \Longleftrightarrow R^{2}-R\left(p_{+}+a+d\right)+p_{+}(a+d)-a b=0 .
$$

Compatibility conditions at $x=0$. We can now proceed to evaluate the coefficients $\alpha_{0}, \alpha_{ \pm}, \beta_{0}$, $\beta_{ \pm}, \gamma_{ \pm}$and $\delta_{ \pm}$related by construction of the exponential forms by

$$
\alpha_{0} P_{0}=a \beta_{0}, \quad \alpha_{ \pm} P_{ \pm}(c)=a \beta_{ \pm}, \quad \gamma_{ \pm} Q_{ \pm}=a \delta_{ \pm} .
$$

With $c$ a free parameter, these leave us 5 coefficients $\alpha_{0}, \alpha_{ \pm}, \gamma_{ \pm}$. For these, we have to write the 5 compatibility conditions at $x=0$, that are

$$
\left\{\begin{array}{l}
u\left(0^{+}\right)=\theta, u\left(0^{-}\right)=\theta, \quad u^{\prime}\left(0^{-}\right)=u^{\prime}\left(0^{+}\right), \\
v\left(0^{+}\right)=v\left(0^{-}\right), \quad v^{\prime}\left(0^{+}\right)=v^{\prime}\left(0^{-}\right) .
\end{array}\right.
$$

They can be rewritten as

$$
\left\{\begin{array}{l}
\alpha_{+}+\alpha_{-}+\alpha_{0}=\theta, \quad \gamma_{+}+\gamma_{-}=1-\theta, \quad \lambda_{+} \alpha_{+}+\lambda_{-} \alpha_{-}+\lambda_{0} \alpha_{0}=\mu_{+} \gamma_{+}+\mu_{-} \gamma_{-}, \\
P_{+} \alpha_{+}+P_{-} \alpha_{-}+P_{0} \alpha_{0}=\frac{a b}{a+d}-Q_{+} \gamma_{+}-Q_{-} \gamma_{-}, \\
\lambda_{+} P_{+} \alpha_{+}+\lambda_{-} P_{-} \alpha_{-}+\lambda_{0} P_{0} \alpha_{0}=\mu_{+} Q_{+} \gamma_{+}+\mu_{-} Q_{-} \gamma_{-} .
\end{array}\right.
$$

After eliminating the terms $\alpha_{0}$ and $\gamma_{+}$using the first two relations, these are

$$
\left\{\begin{array}{l}
\left(\lambda_{0}-\lambda_{+}\right) \alpha_{+}+\left(\lambda_{0}-\lambda_{-}\right) \alpha_{-}-\left(\mu_{+}-\mu_{-}\right) \gamma_{-}=-\mu_{+}(1-\theta)+\lambda_{0} \theta, \\
\left(P_{0}-P_{+}\right) \alpha_{+}+\left(P_{0}-P_{-}\right) \alpha_{-}+\left(Q_{+}-Q_{-}\right) \gamma_{-}=P_{0} \theta-\frac{a b}{a+d}+Q_{+}(1-\theta), \\
\left(\lambda_{0} P_{0}-\lambda_{+} P_{+}\right) \alpha_{+}+\left(\lambda_{0} P_{0}-\lambda_{-} P_{-}\right) \alpha_{-}-\left(\mu_{+} Q_{+}-\mu_{-} Q_{-}\right) \gamma_{-}=\lambda_{0} P_{0} \theta-\mu_{+} Q_{+}(1-\theta) .
\end{array}\right.
$$

In order to analyze if the solutions exist and define positives waves, we can reduce the complexity by considering the large $c$ asymptotic. One can observe a posteriori that the correct expansion is to set

The system becomes

$$
\alpha_{+}=\frac{a_{+}}{c^{2}}, \quad \alpha_{-}=\theta+\frac{a_{-}}{c^{2}} .
$$

$$
\left\{\begin{array}{l}
\frac{\lambda_{0}-\lambda_{+}}{c} a_{+}+\frac{\lambda_{0}-\lambda_{-}}{c} a_{-}-c\left(\mu_{+}-\mu_{-}\right) \gamma_{-}=-c \mu_{+}(1-\theta)+c \lambda_{-} \theta, \\
\frac{P_{0}-P_{+}}{c^{2}} a_{+}+\frac{P_{0}-P_{-}}{c^{2}} a_{-}+\left(Q_{+}-Q_{-}\right) \gamma_{-}=P_{-} \theta-\frac{a b}{a+d}+Q_{+}(1-\theta), \\
\frac{\lambda_{0} P_{0}-\lambda_{+} P_{+}}{c^{3}} a_{+}+\frac{\lambda_{0} P_{0}-\lambda_{-} P_{-}}{c^{3}} a_{-}-\frac{\mu_{+} Q_{+}-\mu_{-} Q_{-}}{c} \gamma_{-}=\frac{\lambda_{-} P_{-}}{c} \theta-\frac{\mu_{+} Q_{+}}{c}(1-\theta) .
\end{array}\right.
$$

Then, thanks to the expansions (16), (17), (19), (20), this is also, with coefficients up to $O\left(\frac{1}{c^{2}}\right)$ according to the expansions at hand

$$
\left\{\begin{array}{l}
\frac{1-\nu}{\nu} a_{+}+\frac{1}{\nu} a_{-}-\left(R_{+}-R_{-}\right) \gamma_{-}=-R_{+}(1-\theta)+\rho_{-} \theta \\
\frac{1-\nu}{\nu^{2}} a_{+}+\frac{1-\nu}{\nu^{2}} a_{-}+\left(R_{+}-R_{-}\right) \gamma_{-}=-\left(\rho_{-}-p_{-}\right) \theta-\frac{a}{a+d}+\left(R_{+}-p_{+}\right)(1-\theta) \\
-\frac{1-\nu}{\nu^{3}} a_{+}-\frac{1-\nu}{\nu^{3}} a_{-}=0 .
\end{array}\right.
$$


The system is invertible, which justifies a posteriori that we can neglect the terms of order $O\left(\frac{1}{c}\right)$, and gives (it is simple to argue reversing the order of the equations)

$$
\left\{\begin{array}{l}
a_{-}=-p_{+}(1-\theta)+p_{-} \theta+\frac{a b}{a+d} \\
\left(R_{+}-R_{-}\right) \gamma_{-}=-\left(\rho_{-}-p_{-}\right) \theta+\frac{a b}{a+d}+\left(R_{+}-p_{+}\right)(1-\theta), \\
a_{+}=-a_{-} .
\end{array}\right.
$$

Consequently, back to the system (21), we conclude that $\alpha_{0}=0\left(c^{-4}\right)$.

Notice that we have

Lemma 3.4 We have the signs

$$
a_{-} \geq \frac{a b}{a+d}>0, \quad \quad \gamma_{-}>0, \quad \quad \mu_{-} \gamma_{-}+\mu_{+} \gamma_{+}>0
$$

Proof. The first inequality follows immediately from (5).

For the second inequality, we have, using the expressions of $\rho_{-}$and $R_{+}$,

$$
\begin{aligned}
\left(R_{+}\right. & \left.-R_{-}\right) \gamma_{-} \\
& =\frac{1}{2}\left[p_{-} \theta-p_{+}(1-\theta)\right]+\frac{a b}{a+d}+\frac{a+d}{2}-\frac{\theta}{2} \sqrt{\left(a+d+p_{-}\right)^{2}+4 a b}+\frac{1-\theta}{2} \sqrt{\left(p_{+}-a-d\right)^{2}+4 a b} \\
& \geq \frac{1}{2}\left[p_{-} \theta-p_{+}(1-\theta)\right]+\frac{a b}{a+d}+\frac{a+d}{2}-\frac{\theta}{2}\left[a+d+p_{-}+\frac{2 a b}{a+d+p_{-}}\right]+\frac{1-\theta}{2}\left(p_{+}-a-d\right) \\
& \geq(1-\theta) \frac{a b}{a+d}>0 .
\end{aligned}
$$

The third inequality follows from the expression (obtained using 22 and $\rho_{-}>p_{-}$)

$$
\begin{aligned}
c\left[\mu_{-} \gamma_{-}+\mu_{+} \gamma_{+}\right] & =R_{+}(1-\theta)-\left(R_{+}-R_{-}\right) \gamma_{-} \\
& =R_{+}(1-\theta)+\left(\rho_{-}-p_{-}\right) \theta-\frac{a b}{a+d}-\left(R_{+}-p_{+}\right)(1-\theta) \\
& >-\frac{a b}{a+d}+p_{+}(1-\theta)>0
\end{aligned}
$$

by assumption (13).

Therefore, we may analyze the monotonicity of $u$.

- For $x>0, u$ is positive because it is a convex combination of the value at $x \gg 1$ (and dominant term is $u \approx \theta e^{-\lambda_{-} x}$ at infinity) and of its value at $x=0$ which is $\theta$. To determine if it is decreasing, we compute the derivative, and for $c \gg 1$, we find

$$
-u^{\prime}(x)=\alpha_{0} \lambda_{0} e^{-\lambda_{0} x}+\alpha_{+} \lambda_{+} e^{-\lambda_{+} x}+\alpha_{-} \lambda_{-} e^{-\lambda_{-} x} \approx-\frac{a_{-}}{c} e^{-\lambda_{+} x}+\frac{\theta \rho_{-}}{c} e^{-\lambda_{-} x}>0,
$$

because it is again a convex combination at $x=0$ and $x \gg 1$, and because $-\frac{a_{-}}{c}+\frac{\theta \rho_{-}}{c}=\mu_{-} \gamma_{-}+\mu_{+} \gamma_{+}>$ 0 thanks to the lemma 3.4

- For $x<0$ the dominant term at $-\infty$ is $1-\gamma_{-} e^{\mu_{-} x}<1$ thanks to lemma 3.4. Also we have

$$
-u^{\prime}(x)=\mu_{-} \gamma_{-} e^{\mu_{-} x}+\mu_{+} \gamma_{+} e^{\mu_{+} x}
$$

and because $-u^{\prime}(x)>0$ for $x \ll-1$ and $-u^{\prime}(0)>0$, we have again $u^{\prime}(x)<0$. 


\section{Bistable nonlinearity}

We consider now the case of a bistable nonlinearity and thus solutions of

$$
\left\{\begin{array}{lc}
-c u^{\prime}-u^{\prime \prime}=f(u)+a v, & u(-\infty)=1, \quad u(\infty)=0, \quad u(0)=\theta \\
-c v^{\prime}-\nu v^{\prime \prime}=u-(a+d) v, & v(-\infty)=\frac{1}{a+d}, \quad v(\infty)=0
\end{array}\right.
$$

with

$$
f(u)=\left\{\begin{array}{l}
-p_{-} u \quad 0 \leq u \leq \theta \\
p_{+}(1-u)-\frac{a}{a+d} \quad \theta \leq u \leq 1
\end{array}\right.
$$

We assume that all numbers $a, b, d, \nu, p_{ \pm}$are positive, $\theta \in(0,1)$ and that the relations hold

$$
\nu<1, \quad p_{-}(a+d)>a, \quad p_{+}(a+d)>a .
$$

We are going to consider condition so as solutions exists with the explicit form

$$
\begin{gathered}
\text { for } x \geq 0 \quad\left\{\begin{array}{l}
u=\alpha_{+} e^{-\lambda_{+} x}+\alpha_{-} e^{-\lambda_{-} x}, \\
v=\beta_{+} e^{-\lambda_{+} x}+\beta_{-} e^{-\lambda_{-} x},
\end{array}\right. \\
\text { for } x \leq 0 \quad\left\{\begin{array}{l}
u=1-\gamma_{+} e^{\mu_{+} x}-\gamma_{-} e^{\mu_{-} x}, \\
v=\frac{1}{a+d}-\delta_{+} e^{\mu_{+} x}-\delta_{-} e^{\mu_{-} x}
\end{array}\right.
\end{gathered}
$$

Theorem 4.1 (Bistable case) We make assumption (25). If a traveling wave of speed $c$ with the above expressions exists, then $\lambda_{ \pm}(c)$ and $\mu_{ \pm}(c)$ should satisfy a nonlinear relation.

There is at least one value of $c^{*}$ such that this relation is satisfied.

It is however unclear if the profile of $u$ obtained with our construction is monotonic. Therefore, we leave as an open problem to check that there are traveling waves associated with these values $c^{*}$. We also leave open the question of uniqueness of $c^{*}$.

The end of this section is devoted to the proof of Theorem 3.1 .

Step 1. The exponential form for $x \geq 0$. The construction uses that all solutions are linear combinations of the form

$$
u=\alpha e^{-\lambda x}, \quad v=\beta e^{-\lambda x} \text { for } x \geq 0
$$

satisfies

$$
\left\{\begin{array}{l}
c \lambda-\lambda^{2}+p_{-}=a \frac{\beta}{\alpha} \\
c \lambda-\nu \lambda^{2}+a+d=\frac{\alpha}{\beta}
\end{array}\right.
$$

This gives the two values $0<\lambda_{-}(c)<\lambda_{+}(c)$, namely the two positive solutions of

$$
\Pi_{+}(\lambda):=\left(c \lambda-\lambda^{2}+p_{-}\right)\left(c \lambda-\nu \lambda^{2}+a+d\right)=a .
$$

Indeed, the fourth order polynomial on the left has two positive roots and two negative roots. The condition (25) tells us that its value at $\lambda=0$ is larger than $a$. We also mention the properties

$$
\left\{\begin{array}{l}
\lambda_{-}(c)<\min \left(\frac{c+\sqrt{c^{2}+4 p_{-}}}{2}, \frac{c+\sqrt{c^{2}+4 \nu(a+d)}}{2 \nu}\right) \\
\lambda_{+}(c)>\max \left(\frac{c+\sqrt{c^{2}+4 p_{-}}}{2}, \frac{c+\sqrt{c^{2}+4 \nu(a+d)}}{2 \nu}\right)
\end{array}\right.
$$




$$
\begin{array}{ccc}
P_{-}(c):=c \lambda_{-}-\lambda_{-}^{2}+p_{-}>0, & P_{+}(c):=c \lambda_{+}-\lambda_{+}^{2}+p_{-}<0, \\
\partial_{c} \lambda_{-}(c)>0, \quad \partial_{c} \lambda_{+}(c)>0, & \lambda_{ \pm}(+\infty)=+\infty, \quad \lambda_{ \pm}(-\infty)=0 .
\end{array}
$$

To make the asymptotic more precise, for the end of this section we specify $\nu<1$ as announced (in the case $\nu>1$ the order between $\lambda_{+}$and $\lambda_{-}$is just reversed),

$$
\left\{\begin{array}{l}
\lambda_{-}(c)=c+\frac{p_{-}}{c}+O\left(\frac{1}{c^{3}}\right), \quad \lambda_{+}(c)=\frac{c}{\nu}+\frac{a+d}{c}+O\left(\frac{1}{c^{3}}\right), \quad \text { for } c \gg 1, \\
P_{-}(c)=O\left(\frac{1}{c^{2}}\right), \quad P_{+}(c)=-c^{2} \frac{1-\nu}{\nu^{2}}+O(1), \quad \text { for } c \gg 1, \\
\lambda_{ \pm}(c)=\frac{\rho_{ \pm}}{c}+O\left(\frac{1}{c^{3}}\right), \quad \rho_{ \pm}=\frac{-\left(a+d+p_{-}\right) \mp \sqrt{\left(a+d-p_{-}\right)^{2}+4 a b}}{2}, \quad \text { for } c \ll-1, \\
P_{ \pm}(c)=p_{-}+\rho_{ \pm}, \quad \text { for } c \ll-1 .
\end{array}\right.
$$

Notice that $\rho_{ \pm}<0$.

Proof of the statement 29]. Monotonicity is a consequence of the equality, along the roots,

$$
\lambda\left[\left(c \lambda-\lambda^{2}+p_{-}\right)+\left(c \lambda-\nu \lambda^{2}+a+d\right)\right]+\partial_{c} \lambda \Pi_{+}^{\prime}(\lambda)=0,
$$

and of the fact that $\Pi_{+}^{\prime}\left(\lambda_{-}\right)<0, \Pi_{+}^{\prime}\left(\lambda_{+}\right)>0$.

The limit at $+\infty$ follows because we know that $\min \left(\frac{c+\sqrt{c^{2}+4 p_{-}}}{2}, \frac{c+\sqrt{c^{2}+4 \nu(a+d)}}{2 \nu}\right)>\min \left(1, \frac{1}{\nu}\right) c$ and for $\lambda=\min \left(1, \frac{1}{\nu}\right) c$ then $\Pi_{+}(\lambda)>a$, therefore $\min \left(1, \frac{1}{\nu}\right) c<\lambda_{-}(c)$.

The limit at $-\infty$ follows using the same type of argument because $\max \left(\frac{c+\sqrt{c^{2}+4 p_{-}}}{2}, \frac{c+\sqrt{c^{2}+4 \nu(a+d)}}{2 \nu}\right) \approx$ $\max \left(p_{-}, a+d\right) \frac{1}{|c|}$ and for $\lambda=\frac{r}{|c|}$ with $r>p_{-}+a+d$ we have $\Pi_{+}(\lambda)>a$ for $c \ll-1$.

Step 2. The exponential form for $x \leq 0$. We may proceed similarly for $x \leq 0$ and the corresponding formulas are

$$
\left\{\begin{array}{l}
-c \mu-\mu^{2}+p_{+}=a \frac{\delta}{\gamma} \\
-c \mu-\nu \mu^{2}+a+d=\frac{\gamma}{\delta} .
\end{array}\right.
$$

The possible bounded solutions are given by the two roots $0<\mu_{-}(c)<\mu_{+}(c)$ of the polynomial

$$
\Pi_{-}(\mu):=\left(-c \mu-\mu^{2}+p_{+}\right)\left(-c \mu-\nu \mu^{2}+a+d\right)=a \text {. }
$$

These roots satisfy

$$
\left\{\begin{array}{l}
\mu_{-}<\min \left(\frac{-c+\sqrt{c^{2}+4 p_{+}}}{2}, \frac{-c+\sqrt{c^{2}+4 \nu(a+d)}}{2 \nu}\right) \\
\mu_{+}>\max \left(\frac{-c+\sqrt{c^{2}+4 p_{+}}}{2}, \frac{-c+\sqrt{c^{2}+4 \nu(a+d)}}{2 \nu}\right)
\end{array}\right.
$$

and

$$
\begin{gathered}
Q_{-}(c):=-c \mu_{-}-\mu_{-}^{2}+p_{+}>0, \quad Q_{+}(c):=-c \mu_{+}-\mu_{+}^{2}+p_{+}<0, \\
\partial_{c} \mu_{-}<0, \quad \partial_{c} \mu_{+}<0, \quad \mu_{ \pm}(+\infty)=0, \quad \mu_{ \pm}(-\infty)=+\infty . \\
\left\{\begin{array}{l}
\mu_{ \pm}(c)=\frac{R_{ \pm}}{c}+O\left(\frac{1}{c^{3}}\right), \quad R_{ \pm}=R_{ \pm}=\frac{p_{+}+a+d \pm \sqrt{\left(p_{+}-a-d\right)^{2}+4 a}}{2}, \quad \text { for } c \gg 1, \\
Q_{ \pm}(c)=p_{+}-R_{ \pm}+O\left(\frac{1}{c^{2}}\right), \quad \text { for } c \gg 1, \\
\mu_{-}(c)=-c-\frac{p_{+}}{c}+O\left(\frac{1}{c^{3}}\right), \quad \mu_{+}(c)=-\frac{c}{\nu}-\frac{a+d}{c}+O\left(\frac{1}{c^{3}}\right), \quad \text { for } c \ll-1, \\
Q_{-}(c)=O\left(\frac{1}{c^{2}}\right), \quad Q_{+}(c)=-c^{2} \frac{1-\nu}{\nu^{2}}+O(1) \quad \text { for } c \ll-1 .
\end{array}\right.
\end{gathered}
$$

Notice that $R_{+} \geq \max \left(p_{+}, a+d\right)$ and $R_{-} \leq \min \left(p_{+}, a+d\right)$. 
Step 3. Compatibility conditions at $x=0$. We can now proceed to evaluate the coefficients $\alpha_{ \pm}, \beta_{ \pm}$, $\gamma_{ \pm}$and $\delta_{ \pm}$related by construction of the exponential forms by

$$
\alpha_{ \pm} P_{ \pm}(c)=a \beta_{ \pm}, \quad \gamma_{ \pm} Q_{ \pm}=a \delta_{ \pm}
$$

These leave us 4 coefficients $\alpha_{ \pm}, \gamma_{ \pm}$and $c$. For these, we have to write the compatibility conditions at $x=0$, that are

$$
\left\{\begin{array}{l}
u\left(0^{+}\right)=\theta, u\left(0^{-}\right)=\theta, \quad u^{\prime}\left(0^{-}\right)=u^{\prime}\left(0^{+}\right) \\
v\left(0^{+}\right)=v\left(0^{-}\right), \quad v^{\prime}\left(0^{+}\right)=v^{\prime}\left(0^{-}\right)
\end{array}\right.
$$

They can be rewritten as

$$
\left\{\begin{array}{l}
\alpha_{+}+\alpha_{-}=\theta, \quad \gamma_{+}+\gamma_{-}=1-\theta, \quad \lambda_{+} \alpha_{+}+\lambda_{-} \alpha_{-}=\mu_{+} \gamma_{+}+\mu_{-} \gamma_{-}, \\
P_{+} \alpha_{+}+P_{-} \alpha_{-}=\frac{a}{a+d}-Q_{+} \gamma_{+}-Q_{-} \gamma_{-}, \quad \lambda_{+} P_{+} \alpha_{+}+\lambda_{-} P_{-} \alpha_{-}=\mu_{+} Q_{+} \gamma_{+}+\mu_{-} Q_{-} \gamma_{-} .
\end{array}\right.
$$

After eliminating the terms $\alpha_{-}$and $\gamma_{-}$using the first two relations, these are

$$
\left\{\begin{array}{l}
\left(\lambda_{+}-\lambda_{-}\right) \alpha_{+}+\lambda_{-} \theta=\left(\mu_{+}-\mu_{-}\right) \gamma_{+}+\mu_{-}(1-\theta) \\
\left(P_{+}-P_{-}\right) \alpha_{+}+P_{-} \theta=\frac{a}{a+d}-\left(Q_{+}-Q_{-}\right) \gamma_{+}-Q_{-}(1-\theta), \\
\left(\lambda_{+} P_{+}-\lambda_{-} P_{-}\right) \alpha_{+}+\lambda_{-} P_{-} \theta=\left(\mu_{+} Q_{+}-\mu_{-} Q_{-}\right) \gamma_{+}+\mu_{-} Q_{-}(1-\theta) .
\end{array}\right.
$$

We now eliminate $\gamma_{+}$using the first equation and find

$$
\left\{\begin{array}{l}
{\left[\frac{P_{+}-P_{-}}{Q_{+}-Q_{-}}+\frac{\lambda_{+}-\lambda_{-}}{\mu_{+}-\mu_{-}}\right] \alpha_{+}=\frac{\frac{a}{a+d}-Q_{-}(1-\theta)-P_{-} \theta}{Q_{+}-Q_{-}}+\frac{\mu_{-}(1-\theta)-\lambda_{-} \theta}{\mu_{+}-\mu_{-}},} \\
{\left[\frac{\lambda_{+} P_{+}-\lambda_{-} P_{-}}{\mu_{+} Q_{+}-\mu_{-} Q_{-}}-\frac{\lambda_{+}-\lambda_{-}}{\mu_{+}-\mu_{-}}\right] \alpha_{+}=\frac{\mu_{-} Q_{-}(1-\theta)-\lambda_{-} P_{-} \theta}{\mu_{+} Q_{+}-\mu_{-} Q_{-}}-\frac{\mu_{-}(1-\theta)-\lambda_{-} \theta}{\mu_{+}-\mu_{-}} .}
\end{array}\right.
$$

The value $c$ is given by the equality

$$
\begin{aligned}
{\left[\frac{P_{+}-P_{-}}{Q_{+}-Q_{-}}+\frac{\lambda_{+}-\lambda_{-}}{\mu_{+}-\mu_{-}}\right] } & {\left[\frac{\mu_{-} Q_{-}(1-\theta)-\lambda_{-} P_{-} \theta}{\mu_{+} Q_{+}-\mu_{-} Q_{-}}-\frac{\mu_{-}(1-\theta)-\lambda_{-} \theta}{\mu_{+}-\mu_{-}}\right] } \\
= & {\left[\frac{\lambda_{+} P_{+}-\lambda_{-} P_{-}}{\mu_{+} Q_{+}-\mu_{-} Q_{-}}-\frac{\lambda_{+}-\lambda_{-}}{\mu_{+}-\mu_{-}}\right]\left[\frac{\frac{a}{a+d}-Q_{-}(1-\theta)-P_{-} \theta}{Q_{+}-Q_{-}}+\frac{\mu_{-}(1-\theta)-\lambda_{-} \theta}{\mu_{+}-\mu_{-}}\right] . }
\end{aligned}
$$

Step 4. Existence of a value c. We re-arrange the above relation as

$$
\mathcal{F}(c):=(1-\theta) A(c)+\theta B(c)-\frac{a}{a+d}\left(\mu_{+}-\mu_{-}\right) C(c)=0 .
$$

With the expressions

$$
\begin{gathered}
C(c)=\left(\lambda_{+} P_{+}-\lambda_{-} P_{-}\right)\left(\mu_{+}-\mu_{-}\right)-\left(\mu_{+} Q_{+}-\mu_{-} Q_{-}\right)\left(\lambda_{+}-\lambda_{-}\right) \\
D(c)=\left(P_{+}-P_{-}\right)\left(\mu_{+}-\mu_{-}\right)+\left(Q_{+}-Q_{-}\right)\left(\lambda_{+}-\lambda_{-}\right) \\
A(c)=\left[Q_{-}\left(\mu_{+}-\mu_{-}\right)-\mu_{-}\left(Q_{+}-Q_{-}\right)\right] C(c)+\mu_{-}\left[Q_{-}\left(\mu_{+}-\mu_{-}\right)-\left(\mu_{+} Q_{+}-\mu_{-} Q_{-}\right)\right] D(c),
\end{gathered}
$$

and thus

$$
A(c)=\left[\mu_{+} Q_{-}-\mu_{-} Q_{+}\right] C(c)-\mu_{-} \mu_{+}\left(Q_{+}-Q_{-}\right) D(c) .
$$




$$
B(c)=\left[P_{-}\left(\mu_{+}-\mu_{-}\right)+\lambda_{-}\left(Q_{+}-Q_{-}\right)\right] C(c)+\lambda_{-}\left[-P_{-}\left(\mu_{+}-\mu_{-}\right)+\left(\mu_{+} Q_{+}-\mu_{-} Q_{-}\right)\right] D(c) .
$$

As general properties, from the signs in the definitions (28) and (33), we discover that

$$
\begin{aligned}
& D(c)<0, \quad \mu_{+} Q_{-}-\mu_{-} Q_{+}>0, \quad \mu_{-} \mu_{+}\left(Q_{+}-Q_{-}\right)<0, \\
& Q_{-}\left(\mu_{+}-\mu_{-}\right)-\mu_{-}\left(Q_{+}-Q_{-}\right)>0 .
\end{aligned}
$$

A comparison among $A(c), B(c)$ and $C(c)$ gives

$$
A(c)<C(c) .
$$

However these properties and monotonicity properties stated earlier do not seem to be enough to conclude a monotonicity property useful for uniqueness. Therefore, to prove existence of a solution of (36), we argue by continuity only and study the expansions for $|c| \gg 1$.

Analysis for $c \gg 1$. We only compute the dominant terms:

$$
\begin{gathered}
C(c) \approx-c^{2} \frac{1-\nu}{\nu^{3}}\left(R_{+}-R_{-}\right) \\
D(c) \approx c\left[-\frac{1-\nu}{\nu^{2}}\left(R_{+}-R_{-}\right)-\left(R_{+}-R_{-}\right) \frac{1-\nu}{\nu}\right]=-c\left(R_{+}-R_{-}\right) \frac{1-\nu^{2}}{\nu^{2}} \\
A(c) \approx \frac{1}{c}\left[R_{+}\left(p_{+}-R_{-}\right)-R_{-}\left(p_{+}-R_{+}\right) C(c)=-c \frac{1-\nu}{\nu^{3}} p_{+}\left(R_{+}-R_{-}\right)^{2}\right. \\
B(c) \approx-c\left(R_{+}-R_{-}\right) C(c)=c^{3}\left(R_{+}-R_{-}\right)^{2} \frac{1-\nu}{\nu^{3}}
\end{gathered}
$$

We conclude that

$$
\mathcal{F}(c) \approx \theta c^{3}\left(R_{+}-R_{-}\right)^{2} \frac{1-\nu}{\nu^{3}} \quad \text { for } c \gg 1 .
$$

And because we assume $\nu<1$, we conclude that

$$
\mathcal{F}(c) \rightarrow+\infty \quad \text { as } c \rightarrow+\infty .
$$

Analysis for $c \ll-1$.

$$
\begin{gathered}
C(c) \approx-c^{2} \frac{1-\nu}{\nu^{3}}\left(\rho_{+}-\rho_{-}\right) \\
D(c) \approx-c\left(\rho_{+}-\rho_{-}\right) \frac{1-\nu^{2}}{\nu^{2}} \\
A(c) \approx-c^{3} \frac{1-\nu}{\nu^{2}} C+c^{4} \frac{1}{\nu} \frac{1-\nu}{\nu^{2}} D=-c^{5} \frac{(1-\nu)^{2}}{\nu^{3}}\left(\rho_{+}-\rho_{-}\right), \\
B(c) \approx O\left(c^{2}\right) .
\end{gathered}
$$

We conclude that

$$
\mathcal{F}(c) \approx-(1-\theta) c^{5} \frac{(1-\nu)^{2}}{\nu^{3}}\left(\rho_{+}-\rho_{-}\right) \quad \text { for } c \ll-1 .
$$

And because $\rho_{+}<\rho_{-}$, we conclude that

$$
\mathcal{F}(c) \rightarrow-\infty \quad \text { as } c \rightarrow-\infty .
$$

Conclusion. By continuity and from the behaviour of $\mathcal{F}$ at $c= \pm \infty$, we conclude from (37) and (38) that there is at least one solution to $\mathcal{F}\left(c^{*}\right)=0$, and the proof of Theorem 4.1 is complete. 


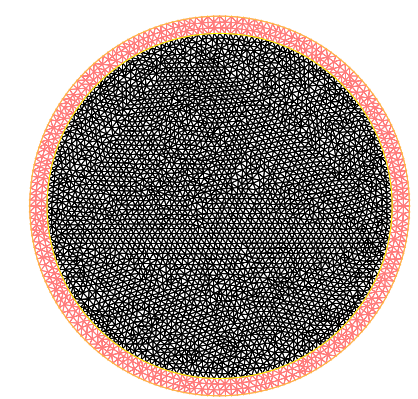

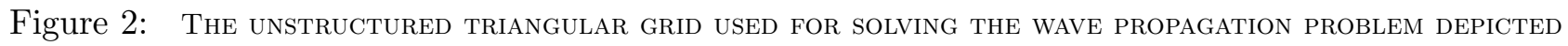
THE FIGURES BELOW.
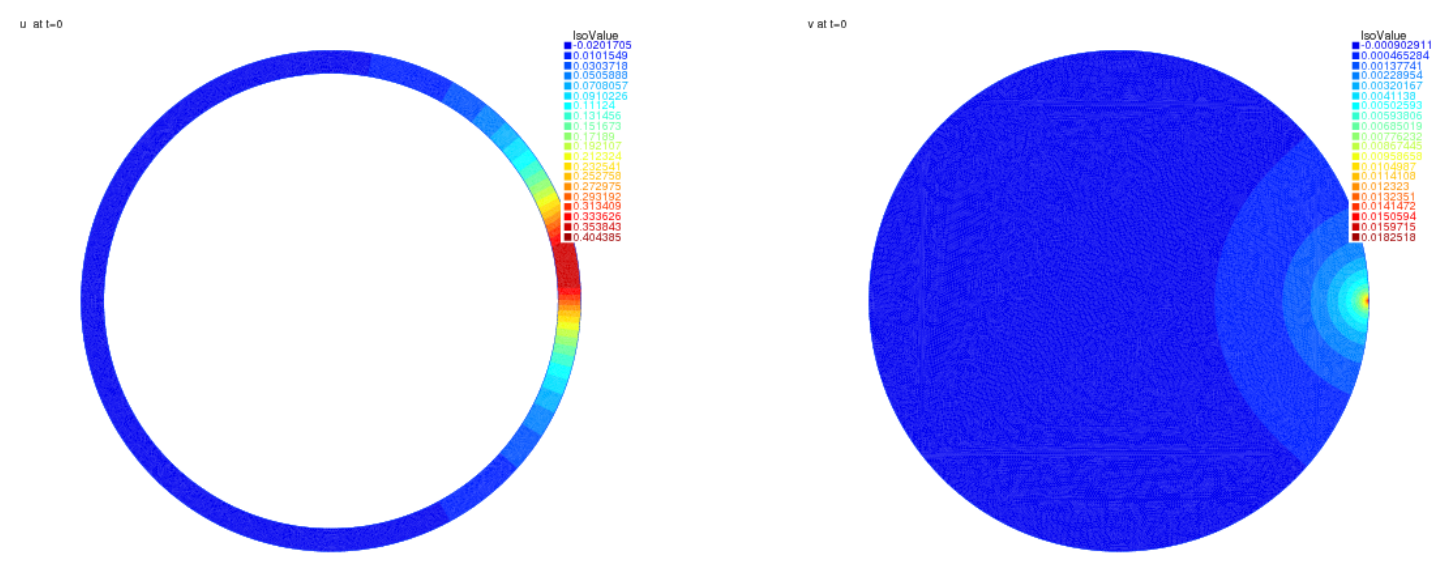

Figure 3: The wave initiation. Left is the 'surface' component $u$. Right is the 'volume' component $v$. See Fig. 4 and Fig. 5 for later times.

\section{$5 \quad$ Numerical simulations}

The coupled systems as (3) and (11), are 'toy models' for the calcic wave initiated after fertilization as mentioned in the introduction.

We present numerical solutions for a reaction-diffusion equation of Fisher-KPP type, propagating on a thin layer around a ball and diffusing within the core of the ball. Except the geometry of the domain which we have chosen to represent a cell and the coupling of $1 \mathrm{D}$ reacting equation to a $2 \mathrm{D}$ diffusion equation arising from the biological problem, this setting is closely related to the problems initiated by [4, 5] and which has led to a very active field of research (see additional references in the introduction).

The numerical simulations has been performed using finite elements and the software FreeFEM++ 9] with an unstructured grid presented in Fig. 2

A wave is initiated (see Fig. 3) at a point located at the right of the computational domain. It propagates a wave propagating along the surface and diffusing in the ball. The Fig. 4 and Fig. 5 present the numerical solution at two different times. One can observe the fast wave propagation compared to the effect of diffusion.

Acknowledgment The authors would like to thank Patrick Cormier for several inspiring discussions on calcic waves which motivated the present study. 

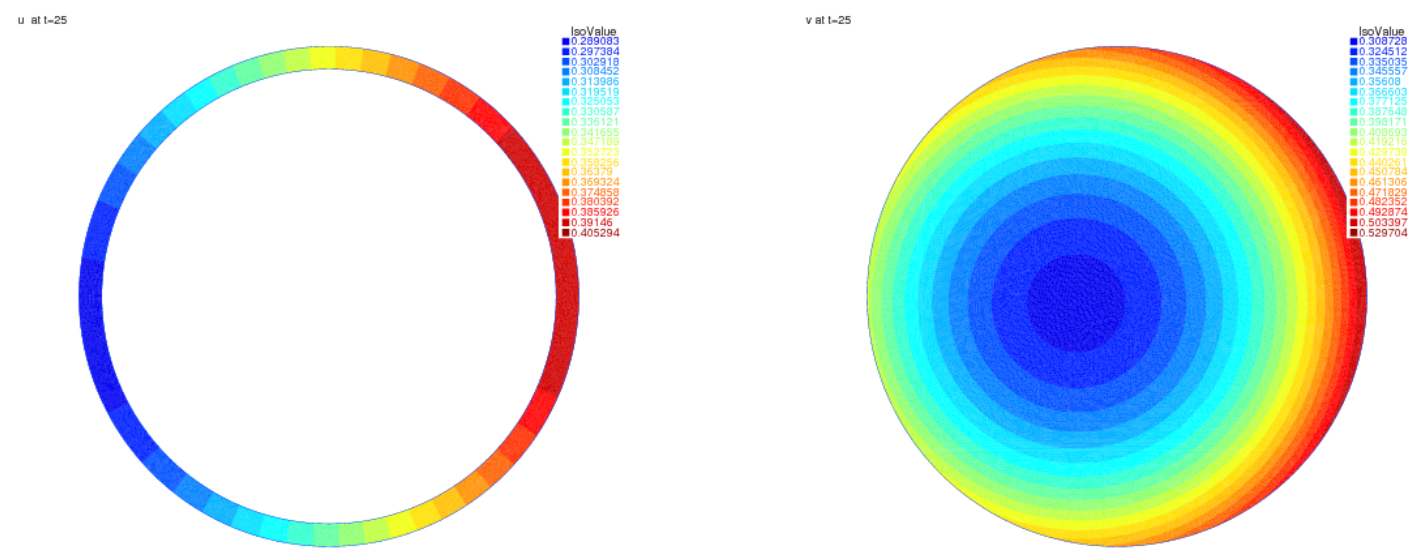

Figure 4: As in Fig. 3 at time $t=25$. The Color scale Changes From left to Right And From one Figure TO THE OTHER.
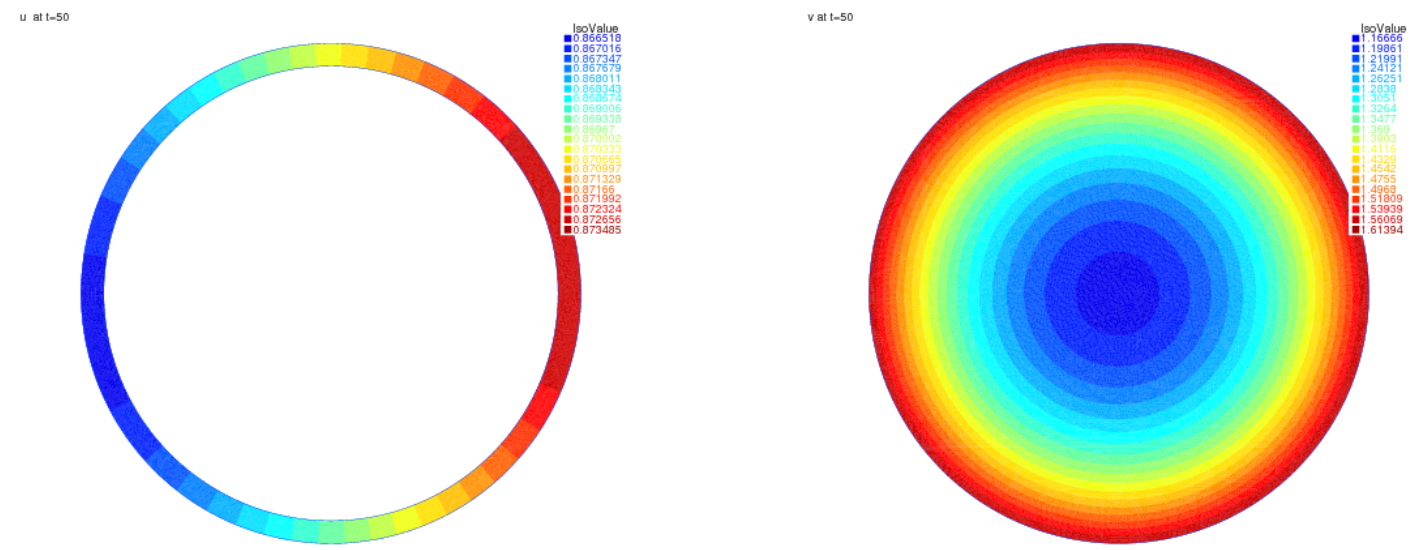

Figure 5: As in Fig. 3 at time $t=50$. The WAVE has invaded the Boundary (COLOR SCALE FROM 0.867 to 0.873 WHILE DIFFUSION IS STILL ACTIVE IN THE BALL (COLOR SCALE FROM 1.17 TO 1.61.

Funding French Cancer League Research Grant (La Ligue contre le Cancer, comités Finistère, Côtes dArmor, Deux-Sèvres et Morbihan); Brittany Regional Council Research Grant (Région Bretagne, project MoDyst) and labex CALSIMLAB for Ph.D. Fellowship (to H.M.).

\section{References}

[1] Henri Berestycki, Anne-Charline Coulon, Jean-Michel Roquejoffre and Luca Rossi. The effect of a line with non-local diffusion on Fisher-KPP propagation. Mathematical Models and Methods in Applied Sciences 25 (2015), 2519-2562.

[2] Henri Berestycki, Anne-Charline Coulon, Jean-Michel Roquejoffre and Luca Rossi. Speed-up of reaction-diffusion fronts by a line of fast diffusion. Séminaire Laurent Schwartz EDP et applications (2013-2014), Exp. No. 19, 25 p., 2013-2014. 
[3] Henri Berestycki, François Hamel, Reaction-diffusion equations and propagation phenomena, Applied Mathematical Sciences, Vol. 600, Springer New York, 2017.

[4] Henri Berestycki, Jean-Michel Roquejoffre and Luca Rossi. The influence of a line with fast diffusion on Fisher-KPP propagation. J. Math. Biol. 66 No. 4-5 (2013), 743-766.

[5] Henri Berestycki, Jean-Michel Roquejoffre and Luca Rossi. Fisher-KPP propagation in the presence of a line : further effects, Nonlinearity, 26 (2013), 2623-2640.

[6] Henri Berestycki, Jean-Michel Roquejoffre and Luca Rossi. The shape of expansion induced by a line with fast diffusion in Fisher-KPP equations. Comm. Math. Phys. 343 (2016), no. 1, 207232.

[7] Henri Berestycki, Jean-Michel Roquejoffre and Luca Rossi. Travelling waves, spreading and extinction for Fisher-KPP propagation driven by a line with fast diffusion.

[8] Laurent Dietrich. Existence of travelling waves for a reactiondiffusion system with a line of fast diffusion. Appl. Math. Res. Express. AMRX 2015, no. 2, 204252.

[9] FreeFEM++, Software available at http://www.freefem.org

[10] Laurent Dietrich. Velocity enhancement of reaction-diffusion fronts by a line of fast diffusion Trans. Amer. Math. Soc. In press.

[11] Léo Girardin and Grégoire Nadin. Travelling waves for diffusive and strongly competitive systems: relative motility and invasion speed. European J. Appl. Math. 26 (2015), no. 4, 521-534.

[12] Lionel F. Jaffe. A proton-led model of fast calcium waves. Cell Calcium 36 (2004) 83-87.

[13] D. C Lane and J. D. Murray. Analysis of Wave Phenomena in a Morphogenetic Mechanochemical Model and an Application to Post-fertilization Waves on Eggs. IMA Journal of Mathematics Applied in Medicine \& Biology (1987) 4, 309-331.

[14] Herbert Levine and Wouter-Jan Rappel. Membrane-bound Turing patterns. Physical Review E $72,0619122(2005)$.

[15] Antoine Pauthier. Two examples of reaction-diffusion front propagation in heterogeneous media. $\mathrm{PhD}$ thesis.

[16] Antoine Pauthier. The influence of nonlocal exchange terms on Fisher-KPP propagation driven by a line of fast diffusion. Commun. Math. Sci. 14 (2016), no. 2, 535-570.

[17] Antoine Pauthier. Uniform dynamics for Fisher-KPP propagation driven by a line of fast diffusion under a singular limit. Nonlinearity 28 (2015), no. 11, 3891-3920.

[18] Benoît Perthame, Parabolic equations in biology. Lecture Notes on Mathematical Modeling in the Life Sciences, Springer (2015). 